\title{
Adaptação transcultural do Chedoke Arm and Hand Activity Inventory (CAHAI) ${ }^{1}$
}

\author{
Daniele Peres, Fernanda Romaguera, Julia Macruz Garcia, Letícia Cardoso Rodrigues, \\ Rodrigo José Knabben, Stella Maris Michaelsen
}

Universidade do Estado de Santa Catarina - UDESC, Florianópolis, SC, Brasil.

\begin{abstract}
Resumo: Introdução: O Chedoke Arm and Hand Activity Inventory (CAHAI) foi desenvolvido nas línguas inglesa e francesa, com versão original nos dois idiomas, para a avaliação do nível de atividade dos membros superiores em indivíduos com hemiparesia após Acidente Vascular Encefálico (AVE). Objetivo: Realizar o processo de adaptação transcultural do manual de aplicação e da folha de pontuação do CAHAI para a língua portuguesa-Brasil. Método: Constituído de sete etapas, o processo de tradução consistiu em: i) duas traduções independentes; ii) compilação das duas traduções, formando uma única tradução; iii) revisão do layout, da tipografia e da gramática; iv) duas retrotraduções independentes; v) reunião com Comitê de Especialistas; vi) envio para a autora da versão original, e vii) pré-teste da versão CAHAI-Brasil (avaliadores: $n=5$; sujeitos: $n=4$ ). Resultados: A versão CAHAI-Brasil teve resultados satisfatórios nas etapas de tradução e adaptação, e índices de concordância entre os avaliadores adequados (kappa entre 0,76 e 1,00). Houve necessidade de substituir alguns termos utilizados no manual e de adaptar alguns dos materiais utilizados no teste. Conclusão: Este estudo mostra que a versão CAHAI-Brasil foi traduzida e adaptada com êxito.
\end{abstract}

Palavras-chave: Tradução, Atividades Cotidianas, Acidente Vascular Cerebral, Extremidade Superior.

\section{Cross-cultural adaptation of the Chedoke Arm and Hand Activity Inventory (CAHAI)}

\begin{abstract}
Introduction: The Chedoke Arm and Hand Activity Inventory (CAHAI) was developed in English and French (original version in both languages) for evaluation of the level of the upper limb activity in subjects with hemiparesis after stroke. Objective: To translate and cross-culturally adapt the manual of application and scoring of CAHAI to Portuguese-Brazil. Method: The process included six steps: the translation process with two independent translation; merging of the two translation; layout, typography and grammar review; two independent backtranslations; meeting with the Committee of Experts, and sending to the author of the original version, and pre-testing of the version CAHAI-Brazil (raters: $n=5$; subjects: $n=4$ ). Results: The CAHAI-Brasil version had satisfactory results in the translation and adaptation, and appropriate index of agreement among raters (kappa between 0,76 and 1,00). Some expressions in the manual and some of the materials used for the test had to be adapted to Brazilian culture. Conclusion: This study show the CAHAI-Brazil version was successfully translated and adapted.
\end{abstract}

Keywords: Translating, Activities of Daily Living, Stroke, Upper Extremity.

Autor para correspondência: Stella Maris Michaelsen, Laboratório de Controle Motor, Universidade do Estado de Santa Catarina, Rua Pascoal Simone, 358, Coqueiros, CEP 88080-350, Florianópolis, SC, Brasil, e-mail: michaelsenstella@hotmail.com

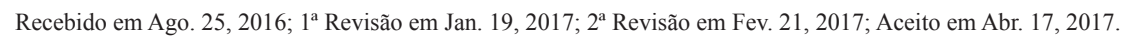




\section{Introdução}

O acidente vascular encefálico (AVE) é uma síndrome neurológica frequente, que engloba comprometimentos motores, como a hemiparesia, em 80\% dos casos (ROGER et al., 2012; BRASIL, 2013). Estima-se que, dos indivíduos que apresentaram paresia severa na fase aguda, $63 \%$ terão comprometimentos residuais, que podem resultar em incapacidade para executar as atividades de vida diária (HUNTER; CROME, 2002). Uma vez que grande parte destas atividades exige a participação de ambos os membros superiores (MMSS) para a sua execuçáo, o comprometimento residual interfere diretamente nas limitaçóes apresentadas pelos indivíduos com hemiparesia no domínio da atividade, de acordo com a Classificação International de Funcionalidade, Incapacidade e Saúde (CIF) (HUNTER; CROME, 2002; LANGHORNE; COUPAR; POLLOCK, 2009; FARIAS; BUCHALLA, 2005).

Instrumentos de avaliação dos MMSS são importantes no processo de reabilitaçáo, norteando o plano de tratamento e fornecendo estimativas realistas da recuperação funcional, para terapeutas, familiares e pacientes (LANGHORNE; COUPAR; POLLOCK, 2009). O Chedoke Arm and Hand Activity Inventory (CAHAI) é um instrumento de avaliação que tem a finalidade de estimar o nível de atividade dos MMSS de indivíduos com hemiparesia, após AVE. Para tanto, 13 tarefas funcionais bilaterais reconhecidas pela Organização Mundial da Saúde (OMS) no domínio de atividade da CIF (BARRECA et al., 2004) são utilizadas para avaliar o envolvimento do membro superior parético (MSP) durante a realização da tarefa.

No entanto, este instrumento foi desenvolvido originalmente nas línguas inglesa e francesa, mas sabe-se que a utilização de instrumentos de avaliação desenvolvidos na língua materna da população-alvo apresenta resultados mais confiáveis. Uma simples tradução do texto para a utilização do instrumento em uma língua diferente da original pode distorcer o significado real de alguns itens, uma vez que os conceitos são influenciados pela cultura local. Isto pode fazer com que alguns aspectos sejam mais relevantes em um país do que seriam em outros (BARRECA et al., 2005; BEATON et al., 2000). Assim, a traduçáo por si é insuficiente para a utilização adequada de um instrumento, justificando a necessidade da realização de um processo estruturado de tradução e adaptação transcultural, de acordo com os aspectos culturais de cada regiāo (BEATON et al., 2000).

A adaptaçáo transcultural de um instrumento é um processo complexo (BEATON et al., 2000).
Este processo aumenta a credibilidade do instrumento na área científica, possibilita a realização de estudos multicêntricos internacionais (GUILLEMIN; BOMBARDIER; BEATON, 1993) e favorece a formação de uma base científica para a comunicação entre profissionais e pesquisadores, assim como a documentação da eficácia de um tratamento (MAHER; LATIMER; COSTA, 2007; SCHUSTER; HAHN; ETTLIN, 2010).

Atualmente, é do nosso conhecimento a existência de dois instrumentos que avaliam a capacidade do MSP disponíveis em português e que incluem tarefas bilaterais. O Teste de Habilidade Motora para o Membro Superior (THMMS) - versão brasileira do AMAT - possui 13 tarefas, das quais seis são bilaterais (cortar carne; abrir a jarra; dar nó em cadarço; colocar o casaco; colocar a camiseta; estender o braço), e avalia a habilidade e a qualidade do movimento. Foi desenvolvido para determinar a eficácia da Terapia de Contensão Induzida (TCI) e não diferencia o membro que executa a tarefa como estabilizador ou manipulador (MORLIN et al., 2006).

O Test d'Évaluation des Membres Supérieurs des Personnes Âgées (TEMPA) é um instrumento que avalia a função dos MMSS em oito tarefas padronizadas, que simulam as AVDs. Das oito tarefas da versão português-Brasil do TEMPA, quatro são bilaterais (abrir um pote e tirar uma colher cheia de café; destrancar uma fechadura, pegar e abrir um recipiente contendo pílulas; escrever em um envelope e colar um selo; embaralhar e distribuir cartas de jogo). Os escores são baseados na velocidade de execução, na graduação funcional e na análise das tarefas. Como o THMMS, este instrumento não diferencia, nas tarefas bilaterais, qual membro realiza a tarefa como manipulador ou como estabilizador, sendo esta limitação identificada pelas autoras (MICHAELSEN et al., 2008).

Considerando-se a importância da capacidade de realizar tarefas bilaterais, é relevante dispormos de instrumentos em língua portuguesa que avaliem essa capacidade. Outros testes de atividade do MS, como ARAT (PAZ; BORGES, 2007), WMFT (PEREIRA et al., 2011), MAS (PEREIRA et al., 2012) e JTT (FERREIRO; SANTOS; CONFORTO, 2010), não apresentam nenhuma tarefa bilateral. O THMMS e o TEMPA não são exclusivamente com tarefas bilaterais e suas cotações não reconhecem o MS que atuou como estabilizador ou como manipulador durante a tarefa, podendo não apresentar uma real evolução do MSP.

Considerando-se que o número de indivíduos com hemiparesia no Brasil é grande e que, no País, têm sido desenvolvidas diversas pesquisas direcionadas 
à reabilitação do MSP, faz-se necessário realizar o processo de adaptação transcultural do CAHAI. Assim, nosso objetivo foi adaptar transculturalmente tanto o manual de aplicação quanto a folha de pontuação do CAHAI, para a língua portuguesa-Brasil.

\section{Método}

Este estudo foi submetido ao Comitê de Ética, sendo por este aprovado (protocolo 131/2010). Todos os participantes desta pesquisa assinaram o Termo de Consentimento Livre e Esclarecido, bem como um Termo de Consentimento para Uso de Fotografias e Vídeos.

\subsection{Instrumento}

O CAHAI foi desenvolvido originalmente nas línguas inglesa e francesa, simultaneamente (BARRECA et al., 2004). A seleção das tarefas que compóem o CAHAI iniciou-se com uma revisão sistemática da literatura, baseada em artigos sobre instrumentos de avaliaçáo do MSP, em indivíduos pós-AVE. Foram compreendidas as bases de dados CINAHL, MEDLINE e EMBASE, e artigos entre os anos de 1960 e 1997. Essa busca resultou em 44 artigos e 21 instrumentos de avaliação. As tarefas funcionais foram definidas como tarefas atuais do cotidiano, tendo sido enumeradas em um total de 171 tarefas funcionais, resultantes dos 21 instrumentos encontrados na busca e que analisam quais tarefas os indivíduos com hemiparesia consideravam relevantes. Além disso, foi realizada uma pesquisa com indivíduos acometidos pelo AVE, para saber quais tarefas são relevantes no seu dia a dia, através de questionamentos, como: "Se seu braço melhorar um pouco, qual atividade você gostaria de fazer?" ou "Quais atividades você pode fazer agora com seu braço e sua mão?”. A partir das respostas, as tarefas foram analisadas, resultando, ao final, em 13 tarefas funcionais bilaterais (BARRECA et al., 2004).

A versão original do CAHAI foi validada pelas autoras que o desenvolveram e este foi aplicado na população com sequelas de AVE, apresentando alta confiabilidade interobservadores, com o CCI de 0,98 [95\% intervalo de confiança (IC), 0,96-0,99]. Fortes correlaçôes foram obtidas entre o CAHAI e os dois instrumentos ARAT e CSMA, quando comparadas à pontuação de dor no ombro do CMSA (1-sided, $\mathrm{P}=0,001)$. As áreas sob as curvas ROC foram: CAHAI, 0,95 (IC 95\%, 0,87-1,00); CMSA, 0,76 (95\% CI, 0,61-0,92), e ARAT, 0,88 (IC 95\%, 0,76 -1,00) (BARRECA et al., 2005).
Em estudo de Schuster et al. (2010), a alta confiabilidade entre avaliadores foi calculada com ICCs para as quatro versóes do CAHAI na versão alemã (CAHAI-G 13, 9, 8, 7), que variam entre $r=0,96$ e $r=0,99(p<0,001)$. Correlação entre a CAHAI-G e as subescalas para mão e braço do CMSA foi de $r=0,74$ ( $p<0,001)$ e $r=0,67$ ( $p<0,001)$, respectivamente. A consistência interna do CAHAI-G para todas as quatro versóes variou entre $\mathrm{a}=0,974$ e $\mathrm{a}=0,979$.

O CAHAI é um instrumento que utiliza a observação da capacidade de indivíduos com hemiparesia, durante a realização de tarefas bilaterais, que refletem atividades de vida diária e, portanto, é uma medida de resultado avaliada objetivamente (MRAO). Diferente de entrevistas ou questionários, em que a medida é baseada no autorrelato do paciente, a MRAO requer que o participante realize tarefas e um examinador, então, determina a pontuação com base em critérios previamente estabelecidos (MORLIN et al., 2006).

De acordo com a Classificação Internacional de Funcionalidade (CIF) (ORGANIZAÇĀO..., 2003), o CAHAI se enquadra no domínio Atividade. Quatro versões do CAHAI foram formadas de acordo com as 13 tarefas que compóem o instrumento: CAHAI -7; CAHAI-8; CAHAI-9 e CAHAI-13. O número subsequente indica o número de atividades estabelecidas em cada versão (BARRECA et al., 2004). Para uma avaliaçáo mais ampla, este estudo utilizou a versáo CAHAI-13, composta de 13 tarefas.

O CAHAI é composto por uma folha de pontuação com uma escala de atividade do MSP, acompanhada de um manual para conceituar a pontuação atribuída pelos avaliadores. Inclui uma tabela de componentes da tarefa, determinando o papel do MSP como estabilizador ou manipulador. Após a observação da função exercida (manipulando ou estabilizando, de acordo com quadro específico no Manual) pelo MSP, durante execução da tarefa, a pontuação é atribuída de acordo com seu grau de independência. A escala de pontuação varia de 1 (quando o MSP executa menos que 25\% de esforço para concluir a tarefa solicitada) a 7 (as atividades são desempenhadas em segurança, sem modificação, sem dispositivos auxiliares ou ajuda, e dentro de um tempo razoável) (BARRECA et al., 2004; SCHUSTER; HAHN; ETTLIN, 2010).

\subsection{Adaptação transcultural}

Diferente da tradução simples, que, em geral, envolve apenas uma pessoa, a adaptação transcultural envolve uma equipe, incluindo tradutores, profissionais da 
saúde que irão utilizar o instrumento e pesquisadores. Este processo envolve a tradução inicial, a síntese, a retrotradução, a revisão pelo comitê de especialistas, o pré-teste e, finalmente, a avaliação das propriedades psicométricas (BEATON et al., 2000; GUILLEMIN; BOMBARDIER; BEATON, 1993; MORLIN et al., 2006; BARRECA et al., 2006; MAHER; LATIMER; COSTA, 2007).

Neste estudo, o processo de adaptação transcultural do manual de aplicação do CAHAI seguiu sete das oito etapas propostas por Beaton et al. (2000). $\mathrm{Na}$ Etapa 4 da versão retrotraduzida por dois tradutores, foi seguida a orientação do estudo de Beaton et al. (2000), segundo a qual deve haver um dos tradutores ingênuo, diferente do proposto por Schuster et al. (2010), que sugerem dois tradutores com conhecimento dos objetivos do estudo. O comitê de Especialistas da Etapa 5 foi composto por quatro fisioterapeutas com experiência em Fisioterapia Neurofuncional. Após a análise da versão retrotraduzida do CAHAI-Brasil pela autora da versão original do CAHAI, foi realizado o pré-teste com a versão brasileira do CAHAI, descrito a seguir. A última etapa, que descreve as propriedades psicométricas do instrumento, deverá ser apresentada em um estudo futuro.

\subsubsection{Pré-teste}

O pré-teste tem o objetivo de avaliar se a adaptação transcultural foi eficiente, para que o instrumento possa ser aplicado sem dúvidas, tanto para o avaliador quanto para o paciente. Além disso, o entendimento dos critérios utilizados para a cotação é a parte que deve estar impecável com relação a traduçáo e adaptação, para que todos avaliadores tenham as mesmas exigências no momento de avaliar o paciente.

O pré-teste foi realizado em três etapas:

Etapa 1 - com cinco fisioterapeutas que aplicaram o CAHAI-Brasil, visando avaliar a clareza do manual quanto a permitir a aplicação do teste e pontuar as tarefas através da observação da capacidade.

Etapa 2 - uma avaliadora participante do processo de traduçáo avaliou quatro pacientes com diferentes comprometimentos motores na fase aguda e na fase crônica, para verificação das dificuldades e teste dos materiais que compóem o "kit" CAHAI-Brasil. A amostra foi definida para ser composta por sujeitos que caracterizassem diferentes tipos de comprometimento motor. Assim, foram recrutados: um participante com comprometimento motor crônico do membro superior leve, um moderado e um severo (de acordo com os três níveis de comprometimento motor da escala de Fugl-Meyer) (MICHAELSEN et al., 2011), e um participante na fase aguda (tempo pós-AVE $\leq 6$ meses), totalizando quatro sujeitos. Os participantes foram recrutados através da Clínica Escola de Fisioterapia do CEFID/ UDESC e do Projeto de Extensão "Atenção à saúde de portadores com sequela de AVE”. Ainda, para a inclusão do indivíduo no estudo, foi estabelecido o critério de pontuação no Mini Exame do Estado Mental (MEEM) sugerido por Brucki et al. (2003), como inferiores a 20, 25, 27, 28 e 29, respectivamente, para analfabetos, 1 a 4,5 a 8,9 a 11, e 12 ou mais anos de escolaridade. Esse instrumento foi realizado para assegurar que o desempenho do indivíduo refletisse totalmente o seu comprometimento motor, sem interferências de possíveis desordens do seu estado mental.

Para caracterização da amostra quanto ao comprometimento motor, à força de preensão e à destreza manual e digital, foram aplicados os seguintes instrumentos, respectivamente: escala de Fugl-Meyer (MICHAELSEN et al., 2011); o dinamômetro de Jamar (BELLACE et al., 2000); Box and Block Test (BBT) (MATHIOWETZ et al., 1985) e Nine Hole Peg Test (NHPT) (OXFORD et al., 2003).

Etapa 3 - duas avaliadoras, uma treinada (AvT - mesma avaliadora da etapa 2 , que teve acesso ao DVD fornecido pela autora do manual original) e outra que utilizou apenas as informaçóes do manual (AvM), aplicaram o CAHAI nos mesmos dois pacientes para identificar eventuais discrepâncias no julgamento da pontuação entre as avaliadoras e eventuais dificuldades de entendimento dos participantes acerca das instruçóes fornecidas pelo avaliador.

Para avaliaçáo da clareza, cinco fisioterapeutas com experiência na área neurofuncional foram convidados a aplicar o CAHAI em um sujeito com hemiparesia e pontuar todas as tarefas, assim como atribuir um conceito quanto à clareza de todos os itens da pontuação: (1) não é claro; (2) um pouco claro; (3) suficientemente claro; (4) altamente claro. Essa etapa possibilitou a verificação da formulaçáo e a adequação dos componentes que compuseram as questóes.

Para a realizaçấo desta etapa, foi encaminhada uma carta, especificando os critérios de avaliação, e a solicitação para apreciação; uma cópia do instrumento proposto para coleta de dados, e uma ficha para avaliação de cada item. Nenhum deles recebeu informaçóes além das constantes no manual em português. Também foi solicitado aos avaliadores anotarem todas as suas dúvidas $\mathrm{e}$ dificuldades durante a aplicação do instrumento. 
Para esta análise, o Índice Kappa foi utilizado para avaliação da concordância entre os avaliadores quanto à clareza de cada uma das tarefas e quanto à clareza de cada item de pontuaçáo de acordo com o conceito atribuído (POLIT et al., 2007).

\section{Resultados}

\subsection{Produção das traduções}

Conforme a primeira etapa do processo de adaptação transcultural, a partir do manual original do CAHAI, foram obtidas duas traduçóes independentes, realizadas por uma fisioterapeuta e por um tradutor sem formação na área da saúde. Ambos nativos da língua portuguesa com conhecimento da língua inglesa, tendo residido em país de idioma inglês. O fisioterapeuta teve conhecimento dos objetivos do estudo e elaborou a versão de tradução chamada $\mathrm{T} 1$, enquanto o tradutor náo teve conhecimento $\mathrm{e}$ elaborou a versão T2. Foi recomendado aos dois tradutores elaborar um relatório durante a tradução com informaçóes que considerassem pertinentes e que contribuíssem, no caso de dúvidas, para as próximas etapas do processo.

\subsection{Compilação das traduções}

Após essa primeira etapa de tradução, as duas versôes traduzidas $\mathrm{T} 1 \mathrm{e}$ T2 foram compiladas em uma única versão T12. As divergências foram checadas por dois fisioterapeutas. $\mathrm{Na}$ falta de consenso entre os termos das traduçóes T1 e T2, para uma mesma palavra, durante a compilação, os tradutores foram consultados (BARRECA et al., 2005).

Na parte do manual de descrição dos materiais para compor o CAHAI-Brasil, os pesquisadores optaram por uma adaptação da descrição dos mesmos, para que os objetos fossem facilmente reconhecidos no Brasil e exercessem a mesma função dos objetos originais do CAHAI.
Alguns itens foram substituídos por outros julgados mais receptíveis na cultura destinada, sofrendo uma adaptação transcultural (Tabela 1).

\subsection{Revisão}

O terceiro passo foi a correção ortográfica e a revisão gramatical e de tipografia, por uma revisora bacharel em língua portuguesa não envolvida no processo de tradução. Esse passo teve o objetivo de corrigir possíveis erros ortográficos e qualquer incoerência das frases. Houve sugestão para a padronização de palavras que se referiam a um mesmo item, se escritas com termos diferentes. Por exemplo, na tarefa "encher um copo com água", às vezes aparecia como 'jarra' e outras vezes como 'jarro'. O mesmo ocorreu com as palavras 'quadro' e 'tabela', se referindo ao mesmo item. Em análise da versão original, foi verificada a mesma ocorrência, o uso de diferentes palavras para um mesmo item, o que explica tal fato presente na versão T12. A revisora decidiu manter a palavra "quadro" ao longo do manual e tal sugestáo foi aceita pelos pesquisadores deste estudo. Poucas frases sofreram mudança de concordância verbal, para uma melhor compreensão na língua portuguesa.

\subsection{Retrotradução}

Após a correção do português, o manual e a folha de cotação foram enviados para a retrotradução. Dois tradutores com língua materna no inglês, mesma língua de origem do instrumento, e sem conhecimento da versão original do manual do CAHAI, realizaram as retrotraduçóes. Nos relatórios, algumas expressôes não ficaram compreensíveis na língua inglesa, após a retrotradução.

Alguns levantamentos foram feitos com relação à mudança de termos para uma mesma expressão, como, por exemplo, a "escala de atividades de sete pontos", algumas vezes aparecia como a "tabela de atividades de sete pontos", como observado na etapa

Tabela 1. Descrição das palavras substituídas após mescla de T1 e T2.

\begin{tabular}{llll}
\hline \multicolumn{1}{c}{ Página do Manual } & \multicolumn{1}{c}{ Item do Manual } & $\begin{array}{c}\text { Palavra original obtida } \\
\text { da tradução }\end{array}$ & \multicolumn{1}{c}{ Palavra substituída } \\
\hline $\begin{array}{l}\text { Durante todo o Manual } \\
\text { Página } 05\end{array}$ & $\begin{array}{l}\text { Pontuação } \\
\text { Pontuação }\end{array}$ & $\begin{array}{l}\text { Afetado } \\
\text { Aparelhos auxiliares }\end{array}$ & $\begin{array}{l}\text { Parético } \\
\text { Página } 07\end{array}$ \\
Material Necessário & Prato de Melanina auxiliares \\
Página 07 & Material Necessário & Massa de vidraceiro & Prato de plástico duro \\
Página 07 & Material Necessário & Poncho de lã & Massa de modelar \\
Página 07 & Material Necessário & Degraus normais & Degraus padrão \\
Durante todo o Manual \\
Página 11 & Vários itens de tarefas & Mão sobre mão & "Mão sobre mão" \\
Páginas 23 e 24 & Pontuação 2 & Ouvido & Próximo ao ouvido \\
\hline
\end{tabular}


anterior, quando a profissional da língua portuguesa também sugeriu mudanças de algumas palavras. Há uma tabela com a descrição de cada item de pontuação e os critérios que devem ser considerados, de acordo com a respectiva tarefa. A tradutora também sugere, dentro do item "materiais requeridos", que seja especificado o tipo de óculos - em vez de apenas glasses, passar a ser eyeglasses, que seria referente a óculos de grau, especificação não necessária na língua portuguesa.

O segundo tradutor sugeriu que em vez de usarmos o termo "engrossador", traduzido para "thickener", seja usado o "material engrossador de cabo", traduzido por "thickener, the length of the utensil". Tal modificação é devida ao uso do termo apenas para o utensílio garfo utilizado na tarefa "cortar massa de modelar" e servindo para aumentar a espessura do cabo do garfo, com o propósito de facilitar a preensão no cabo durante a realização da tarefa.

\subsection{Comitê de especialistas}

A comissão de especialistas foi composta por quatro fisioterapeutas com experiência na área neurofuncional, que se encontraram para discutir as divergências entre as traduçôes T12, B1 e B2. Ao final, dois encontros foram necessários para a discussão de todo o manual do CAHAI e sua folha de cotação. Um primeiro encontro, com duraçáo de uma hora e meia, para a comparação e análise da primeira parte do manual, e um segundo com duração de duas horas e meia, finalizando a comparaçáo e análise, a partir da descrição da cotação da primeira tarefa até o final do manual.

A comissão de especialistas se preocupou com a escolha da melhor palavra ou termo diante da divergência de palavras nas diferentes traduçóes, baseando-se na versão original do instrumento e buscando uma equivalência conceitual entre os idiomas inglês e português.
Para que todos os comentários dos tradutores e revisores fossem avaliados, foram impressas a versão original, a versão T12 da tradução para a língua portuguesa, a versão com correção de layout, tipografia e gramática, e as versôes retrotraduzidas. Iniciou-se pelas consideraçóes feitas nos relatórios de retrotradução, já analisando as alterações realizadas pela revisora de português, comparando também a retrotradução com a versão original do manual (Tabela 2).

As palavras e frases que o comitê julgou inapropriadas ou de má compreensão na língua portuguesa foram reescritas mesmo não apresentando divergências entre a versão original com a retrotradução.

\subsection{Análise da autora da versão original}

Depois de realizadas as traduções e retrotraduçôes, e a análise do comitê, a versão em português foi originada e traduzida novamente para o inglês. A versão final em português e a sua retrotradução foram enviadas para a autora da versão original do CAHAI, para sugestóes e críticas, assim como para sua aprovação. Uma carta anexa explicou o porquê da troca de alguns termos, como, por exemplo, "client" da versão original, que foi traduzida por "paciente” para a versão em português, por entender que, no Brasil, a palavra "cliente" dá uma conotação comercial ao instrumento.

A autora fez mínimas colocaçóes, sendo que nenhuma destas comprometeu o sentido da versão CAHAI-Brasil, não sendo necessário fazer retorno a quaisquer das etapas anteriores, tendo sido então aprovada a versão em português.

\subsection{Pré-teste}

Na Etapa 1 do pré-teste, foram convidados cinco fisioterapeutas para aplicar o CAHAI-Brasil e avaliar as tarefas e os itens da pontuação, e anotar

Tabela 2. Exemplos de diferenças encontradas e a decisão tomada pela comissão de qual palavra ou termo mais adequado.

\begin{tabular}{|c|c|c|c|c|}
\hline Página do Manual & Versão Original & $\begin{array}{l}\text { Versão do Manual } \\
\text { Traduzido (T12) }\end{array}$ & $\begin{array}{c}\text { Versão } \\
\text { Backtranslation } \\
\text { B1 e B2 }\end{array}$ & $\begin{array}{l}\text { Decisão da } \\
\text { Comissão }\end{array}$ \\
\hline 2 & Stroke & Derrame & Stroke & $\begin{array}{c}\text { Acidente Vascular } \\
\text { Encefálico }\end{array}$ \\
\hline 3 & Scale & Tabela & Table & Escala \\
\hline 4 & Completed & Completados & Completed & Foram concluídos \\
\hline 7 & Pull-on vest & Vestimenta & Clothing & Poncho de tecido \\
\hline 17 & Requires & Necessita de & Requires & Requer \\
\hline Em algumas tarefas & Grasp & Pega & Grip & Preensão \\
\hline
\end{tabular}


as dúvidas quanto à aplicação com o paciente. Os avaliadores eram formados havia mais de três anos, distribuindo-se assim: um avaliador cursando mestrado (avaliador 1 - Av1), um avaliador mestre (avaliador 2 - Av2), dois avaliadores cursando doutorado (avaliador 3 - Av3 e avaliador 4 - Av4) e um avaliador doutor (avaliador 5 - Av5), todos atuantes na área de fisioterapia neurofuncional e com experiência em pacientes pós-AVE.

A Av1 encontrou dificuldade em avaliar o paciente na tarefa 1 , quando o paciente realiza a tarefa com os MMSS apoiados sobre a mesa; segundo o manual, não se enquadra na cotação de número 7 , mas também não se enquadra na cotaçáo de número 6 e 5, ficando uma pontuação bem abaixo do que o paciente realmente poderia receber. Ainda a Avl teve dúvidas em definir a veste poncho utilizada nas tarefas 6 e 10. Segundo ela, em sua região, esse termo não é conhecido. A Av3 teve dúvida na tarefa 5 , para a qual o manual não deixa claro se o pano deve ser totalmente torcido ou uma única vez. A mesma avaliadora teve dificuldades em cotar a tarefa 6 , pois o paciente foi capaz de abotoar os cinco botóes, mas utilizou o MSP como estabilizador, que quase não participou da tarefa. A Av3 também comenta que, nas instruções da tarefa 9, não está explícito que o paciente deve separar os pedaços da massa cortados. A Av4 considerou que, nas tarefas 1, 2, 3, 5, 8, 9 e 11, poderia constar que não se devem apoiar os braços e/ou cotovelos na mesa. $\mathrm{Na}$ tarefa 4, não há especificação se a jarra deve estar completamente cheia. $\mathrm{Na}$ tarefa 7 , a Av4 sugere que pode ser dado, como instrução ao paciente, "secar as costas completamente em ambos os lados", assim como na tarefa 13 , para constar que o paciente deve segurar com a outra máo na barra para subir as escadas. A Av5 não teve comentários sobre os itens das tarefas e apenas ressaltou ficar em dúvida nas tarefas 12 e 13, quando o paciente for cadeirante e não puder realizar a tarefa (Tabela 3A).

Todos os itens alcançaram índices de clareza adequados, em todas as tarefas analisadas. Os itens 1,4 a 7 e 9 foram avaliados com escores inferiores apenas por um avaliador e, desta forma, foi julgado como não comprometendo a clareza de forma geral.

$\mathrm{Na}$ avaliação dos itens da cotação, cujas notas estão descritas na tabela a seguir (Tabela 3B), o Av2 questionou o fato de não constar, na folha de cotação, um espaço para indicar quando o membro parético não atua durante a tarefa. A Av3 diz que a redação está clara, mas no manual não fica claro que a pontuaçáo se refere aos componentes da tarefa, o que poderia ser melhorado com um quadro em anexo

Tabela 3. Resultado da Clareza A: das tarefas e B: dos itens da cotação.

\begin{tabular}{|c|c|c|c|c|c|c|}
\hline \multirow{2}{*}{ ITENS } & \multicolumn{5}{|c|}{ Pontuação } & \multirow{2}{*}{ Kappa } \\
\hline & Av1 & Av2 & Av3 & Av4 & Av5 & \\
\hline \multicolumn{7}{|l|}{ A - Tarefas } \\
\hline 1. Abrir um vidro de café solúvel & 2 & 3 & 4 & 3 & 4 & 0.76 \\
\hline 2. Discar 192 & 4 & 4 & 4 & 3 & 4 & 1.00 \\
\hline 3. Traçar uma linha reta com uma régua & 4 & 4 & 4 & 3 & 4 & 1.00 \\
\hline 4. Encher um copo de água & 4 & 3 & 4 & 2 & 4 & 0.76 \\
\hline 5. Torcer um pano & 4 & 4 & 2 & 3 & 4 & 0.76 \\
\hline 6. Abotoar cinco botões & 4 & 4 & 2 & 4 & 4 & 0.76 \\
\hline 7. Enxugar as costas com toalha & 4 & 4 & 4 & 2 & 4 & 0.76 \\
\hline 8. Colocar creme dental na escova & 4 & 3 & 4 & 3 & 4 & 1.00 \\
\hline 9. Cortar massa de modelar & 4 & 3 & 2 & 3 & 4 & 0.76 \\
\hline 10. Fechar zíper & 4 & 4 & 4 & 4 & 4 & 1.00 \\
\hline 11. Limpar os óculos & 4 & 4 & 3 & 3 & 4 & 1.00 \\
\hline 12. Colocar caixa sobre a mesa & 4 & 4 & 4 & 4 & 4 & 1.00 \\
\hline \multicolumn{7}{|l|}{ B - Pontuação } \\
\hline 1. Assistência total (MSP >25\%) & 3 & 4 & 4 & 3 & 4 & 1.00 \\
\hline 2. Assistência máxima (MSP=25\% - 49\%) & 3 & 4 & 4 & 3 & 4 & 1.00 \\
\hline 3. Assistência moderada ( $\mathrm{MSP}=50 \%-74 \%$ ) & 3 & 4 & 4 & 3 & 4 & 1.00 \\
\hline 4. Assistência mínima (MSP >75\%) & 3 & 4 & 4 & 3 & 4 & 1.00 \\
\hline 5. Supervisão & 3 & 4 & 4 & 3 & 4 & 1.00 \\
\hline 6. Independência modificada (auxílio técnico) & 3 & 4 & 4 & 3 & 4 & 1.00 \\
\hline 7. Independência completa (em tempo normal, em segurança) & 3 & 4 & 4 & 3 & 4 & 1.00 \\
\hline
\end{tabular}

4 = Altamente claro; 3 = Suficientemente claro; 2 = Um pouco claro; 1 = Não é claro. 
a cada tarefa, com os componentes das açóes que devem ser realizadas. O indivíduo pode conseguir realizar a tarefa de forma completa, mas se o MSP não fizer determinada ação, isso já caracteriza assistência, o que não está claro no manual. A Av4 fala que a pontuação não é totalmente clara sem o auxílio do manual para a pontuação de cada tarefa, ou seja, apenas com a folha de pontuação, a clareza fica comprometida.

As sugestóes e dificuldades relatadas pelos avaliadores da clareza foram analisadas e os itens reformulados, porém estas não foram suficientes para alterar o entendimento das instruçôes e da pontuação. Todos os itens da cotação foram avaliados como altamente claros ou suficientemente claros, com a concordância entre os avaliadores apresentando um Kappa = 1,00. Na Etapa 2, participaram do estudo quatro indivíduos com hemiparesia, avaliados pela mesma avaliadora. Os indivíduos eram representativos dos diferentes níveis de comprometimento motor (incluindo a cronicidade) característicos da sequela de AVE, com média de idade de $51 \pm 14,3$ anos, sendo três homens e uma mulher. A avaliação do comprometimento motor, da força de preensão e da destreza manual e digital está descrita na Tabela 4. O tempo de duração da aplicação do CAHAI foi de aproximadamente 30 minutos.

Nessa etapa, a pontuação dada pela avaliadora treinada (AvT) e pela avaliadora somente com acesso ao manual (AvM) está descrita na Tabela 5. Predominantemente, a AvT considerou os pacientes como necessitando de assistência mínima a moderada, enquanto que a AvM considerou os mesmos pacientes como tendo independência modificada ou completa. As tarefas com pontuaçóes mais discrepantes foram as tarefas "torcer um pano" e "cortar massa de modelar"; o item "subir escada com uma sacola" foi avaliado pela AvT em ambiente externo; entretanto, como náo existia escada dentro do ambiente de avaliaçáo, a AvM julgou não pertinente sair do ambiente interno e não avaliou este item.

Tabela 4. Descrição da caracterização dos participantes.

\begin{tabular}{|c|c|c|c|c|c|c|c|}
\hline Sujeito & Sexo & $\begin{array}{l}\text { Tempo } \\
\text { pós-AVE } \\
\text { (meses) }\end{array}$ & $\begin{array}{c}\text { Nível de } \\
\text { comprometimento } \\
\text { motor (EFM-MS) }\end{array}$ & $\begin{array}{c}\text { Força de } \\
\text { preensão } \\
\text { manual } \\
\text { NP/P (kgf) }\end{array}$ & $\begin{array}{c}\text { Destreza } \\
\text { manual (BBT) } \\
\text { NP } / \mathbf{P} \\
\text { (blocos } / \text { min) }\end{array}$ & $\begin{array}{c}\text { Destreza } \\
\text { digital } \\
\text { (NHPT) } \\
\text { NP/P } \\
\text { (seg) }\end{array}$ & CAHAI \\
\hline 1 & M & 1 & Leve $(55 / 66)$ & $34 / 16$ & $52 / 34$ & $64 / 120$ & 68 \\
\hline 2 & M & 18 & Leve $(60 / 66)$ & $36 / 32$ & $50 / 27$ & $30 / 78$ & 77 \\
\hline 3 & $\mathrm{~F}$ & 15 & Moderado (39/66) & $21 / 0$ & $42 / 15$ & $44 / 120$ & 31 \\
\hline 4 & M & 20 & Grave $(29 / 66)$ & $52 / 10$ & $48 / 0$ & $72 / 120$ & 15 \\
\hline
\end{tabular}

$\mathrm{NP}=$ não parético; $\mathrm{P}$ = parético; $\mathrm{seg}$ = segundos; $\mathrm{kgf}$ = quilograma-força; blocos $/ \mathrm{min}$ = quantidade de blocos que o indivíduo pegou e transportou durante um minuto.

Tabela 5. Média das notas dos dois pacientes avaliados por cada avaliadora.

\begin{tabular}{lcc}
\hline \multicolumn{1}{c}{ Tarefa } & Total AvT & Total AvM \\
\hline & Média (DP) & Média (DP) \\
1. abrir o pote & $3,0(0,0)$ & $4,0(0,0)$ \\
2. discar 192 & $3,5(0,7)$ & $6,5(0,7)$ \\
3. traçar uma linha & $3,5(0,7)$ & $4,0(0,0)$ \\
4. encher um copo de água & $2,0(0,0)$ & $5,0(2.8)$ \\
5. torcer um pano & $3,5(0,7)$ & $7,0(0,0)$ \\
6. abotoar cinco botões & $4,0(1,4)$ & $4,5(3,5)$ \\
7. enxugar as costas com toalha & $4,0(1,4)$ & $4,0(0,0)$ \\
8. colocar creme dental na escova & $4,0(0,0)$ & $5,0(2,1)$ \\
9. cortar massa de modelar & $2,5(0,7)$ & $6,5(0,7)$ \\
10. fechar zíper & $3,5(2,1)$ & $6,5(0,7)$ \\
11. limpar os óculos & $4,0(1,4)$ & $6,5(0,7)$ \\
12. colocar caixa sobre a mesa & $4,0(0,0)$ & $6,5(0,7)$ \\
13. subir escada com uma sacola & $4,0(0,0)$ & $\mathrm{NA}$ \\
Total & $45,5(6,4)$ & $66,5(12,0)$ \\
\hline
\end{tabular}

AvT $=$ Avaliador Treinado; AvM = Avaliador com acesso ao Manual; NA= não avaliado. 


\section{Discussão}

Muitos instrumentos de avaliação de indivíduos com hemiparesia são originados em outras línguas e, em sua maioria, desenvolvidos na língua inglesa (REICHENHEIM; MORAES, 2007). A utilização de instrumentos sem um processo de tradução e adaptação transcultural torna duvidosos os resultados de pesquisas que se utilizem desses instrumentos. A cultura e a linguística são divergentes entre os países, até mesmo dos que se utilizam de um mesmo idioma (REICHENHEIM; MORAES, 2007; STREINER; NORMAN, 2003; MICHAELSEN et al., 2011). Isso acarreta expressôes e conceitos que podem não conseguir expressar fatos em um dado idioma. Por isso, é necessário que os instrumentos sejam adaptados transculturalmente, mesmo que este processo não seja tão simples. Adaptar um instrumento culturalmente consiste muito além de uma tradução apenas (BARRECA et al., 2005; MORLIN et al., 2006; REICHENHEIM; MORAES, 2007; MICHAELSEN et al., 2011), há de se analisar todo o conteúdo e identificar o quão relevantes são os aspectos para a população-alvo.

Este estudo realizou a adaptação transcultural do CAHAI para a língua portuguesa seguindo as sete primeiras etapas propostas na literatura (BARRECA et al., 2005; BEATON et al., 2000; MORLIN et al., 2006). A avaliação das propriedades psicométricas será realizada em estudo futuro. Todas as etapas foram realizadas na sequência, algumas com dificuldades, como por exemplo, a etapa da retrotradução, em que a literatura solicita dois retrotradutores com inglês como língua materna e conhecimento na língua portuguesa. Houve dificuldades para encontrar dois retrotradutores seguindo os critérios especificados na literatura, além dos altos custos cobrados por lauda, uma vez que foram realizadas as etapas de tradução e adaptação transcultural tanto pra a folha de cotação quanto para o manual de aplicação e pontuação.

A última etapa realizada por este estudo, o pré-teste, procurou analisar a compreensão da aplicaçáo do instrumento como o entendimento para realização da tarefa por parte do paciente. Também foi observada a facilidade na aquisição dos materiais necessários para aplicaçáo do CAHAI, não havendo dificuldades.

Houve adaptaçôes nos materiais utilizados, como o washcloth, que, em uma tradução literal, seria "pano para o banho". Como este "pano para banho" é pouco utilizado em nossa cultura, substituímos por pequeno pano de limpeza, o que reflete mais adequadamente o material utilizado no vídeo de demonstração e também facilita o entendimento de qual material exatamente será preciso para aquela tarefa. Isso foi possível porque este estudo não se limitou a fazer a adaptação transcultural somente da folha de cotaçáo do instrumento, mas de todo o manual. O que se observa em estudos de tradução e adaptaçáo transcultural de instrumentos que avaliam a populaçáo com hemiparesia pós-AVE é que nem todos citam a tradução do manual de aplicação (CONTE et al., 2009).

No estudo de Michaelsen et al. (2008), os autores traduziram e adaptaram o instrumento Test d'Évaluation des Membres Supérieurs des Personnes Âgées (TEMPA). Este instrumento avalia a quantidade e qualidade de uso dos MMSS paréticos na população pós-AVE. Apesar de os autores não terem seguido todos os passos propostos por Beaton et al. (2000), tiveram a preocupação de analisar e excluir a tarefa de "colocar um cachecol", avaliando não ser uma tarefa adequada para a populaçáo brasileira, já que nosso clima é tropical. Diferente dos achados no estudo de Michaelsen et al. (2008), em nosso estudo todas as tarefas do CAHAI parecem ser significativas para a população brasileira.

Muitos aspectos citados ilustram a complexidade do processo de adaptação transcultural. Pesquisadores normalmente consideram este processo trabalhoso e acabam não compreendendo a importância dessa adaptação, optando pelo uso de instrumentos não traduzidos (BARRECA et al., 2005; GUILLEMIN; BOMBARDIER; BEATON, 1993; MORLIN et al., 2006; BARRECA et al., 2006). Dessa forma, a utilização de instrumentos não adaptados para a população que se deseja estudar apresentará resultados que não identificam aquela populaçáo adequadamente com vieses não identificáveis (GUILLEMIN; BOMBARDIER; BEATON, 1993; MORLIN et al., 2006; BARRECA et al., 2006), divulgação de resultados errôneos e perda de tempo. Estes aspectos evidenciam a complexidade do processo de tradução e adaptação, que deve ser rigoroso, e o torna fundamental para uma pesquisa responsável. Mesmo que seja um processo complexo, ainda é mais fácil do que o desenvolvimento de um novo instrumento (BARRECA et al., 2006).

No pré-teste, os avaliadores tiraram suas conclusōes quanto à aplicação do CAHAI e pontuaram os itens das tarefas e da pontuação. Praticamente todas as dúvidas que vieram dos avaliadores durante a aplicação do CAHAI-Brasil são explicadas no DVD do manual original, tanto as de pontuação como as de aplicação da tarefa. No entanto, ainda não há disponível o DVD do manual em português e os avaliadores do pré-teste não tiveram acesso ao DVD 
do manual em inglês, uma vez que o objetivo do estudo foi verificar a clareza da tradução do manual. Vale ressaltar que, como a Av2 concluiu, o manual está claro, porém alguns itens não são explicados detalhadamente. Nesse sentido, a confecção de um DVD em português, de acordo com as informaçóes contidas no DVD original, seria uma boa soluçáo, como também poderia haver notas de rodapé no próprio manual, como pedem alguns dos comentários feitos pelos avaliadores (por exemplo, uma nota no rodapé definindo "poncho").

Feito o teste-reteste com dois pacientes, notou-se uma grande diferença na pontuaçáo dos pacientes por AV1 e AV2. Nos dois pacientes, a AV2 foi menos exigente, atribuindo notas superiores às notas da AV1. Mesmo seguindo todas as etapas propostas para a tradução e adaptação de instrumentos, no pré-teste, houve diferenças entre a avaliação do avaliador que assistiu ao DVD enviado pelas autoras e a cotação da avaliadora que apenas leu a versão traduzida do manual. Somente a tradução do manual náo deixou claro o nível de exigência motora para a realização das tarefas pelos pacientes. A avaliadora que não assistiu ao DVD tendeu a atribuir uma maior cotaçáo aos pacientes do que a avaliadora que teve acesso ao DVD. Nota-se, mais uma vez, a necessidade de ser confeccionado um DVD em português (BARRECA et al., 2004; SCHUSTER; HAHN; ETTLIN, 2010).

O tempo gasto para aplicação do CAHAI-Brasil foi, em média, o tempo estipulado pelas autoras do CAHAI original, de aproximadamente 30 minutos (BARRECA et al., 2004; SCHUSTER; HAHN; ETTLIN, 2010).

Na maioria dos indivíduos, esse tempo variou em cerca de 15 minutos, para indivíduos com hemiparesia leve, e alcançou uma hora para indivíduos com comprometimento motor severo. O tempo também variou de acordo com a importância que o paciente atribuiu para a utilizaçáo do MSP, sendo que alguns indivíduos com comprometimento severo não tentaram utilizar o MSP, realizando todas as tarefas com a outra mão e levando menos tempo que alguns indivíduos com comprometimento moderado, que faziam questáo de conseguir utilizar o membro parético durante a tarefa.

\section{Conclusão}

Os resultados desta pesquisa mostraram que o processo de adaptação transcultural do manual de aplicação e da folha de pontuação do CAHAI para a língua portuguesa-Brasil foi realizado de acordo com as etapas pré-estabelecidas; entretanto, outras propriedades psicométricas necessitam ser avaliadas para sua aplicação na população brasileira. Não houve nenhum conflito nos termos da traduçáo que não pode ser discutido e resolvido junto com o comitê de especialistas, o que reforça a importância de cada etapa cumprida.

\section{REFERÊNCIAS}

BARRECA, S. et al. Development of the Chedoke Arm and Hand Activity Inventory: theorical constructs, item generation and selection. Topics in Stroke Rehabil, Frederick, v. 11, n. 4, p. 31-42, 2004.

BARRECA, $S$. et al. Test-retest reliability, validity and sensitivity of the Chedoke Arm and Hand Activity Inventory: a new measure of upper-limb function for survivors of stroke. Archives of Physical Medicine and Rehabilitation, Chicago, v. 86, n. 8, p. 1616-1622, 2005.

BARRECA, $S$. et al. Comparing 2 versions of the Chedoke Arm and Hand Activity Inventory with the Action Reseach Arm Test. Physical Therapy, New York, v. 86, n. 2, p. 245-252, 2006.

BEATON, D. E. et al. Guidelines for the process of cross-cultural adaptation of self-report measures. Spine, Philadelphia, v. 25, n. 24, p. 3186-3191, 2000.

BELLACE, J. V. et al. Validity of the dexter evaluation system's Jamar dynamometer attachment for assessment of hand grip strength in a normal population. Journal of Hand Therapy: Official Journal of the American Society of Hand Therapists, Philadelphia, v. 13, n. 1, p. 46-51, 2000.

BRASIL. Ministério da Saúde. Taxa de mortalidade especifica por doenças do aparelho circulatório: Brasil. Brasília: Datasus, 2013. Disponível em: <http://tabnet.datasus. gov.br/tabdata/LivroIDB/2edrev/c08.pdf>. Acesso em: 15 mar. 2015.

BRUCKI, S. M. D. et al. Sugestóes para o uso do Mini-Exame do Estado Mental no Brasil. Arquivos de Neuropsiquiatria, São Paulo, v. 61, n. 3B, p. 777-781, 2003.

CONTE, A. L. F. et al. Confiabilidade, compreensão e aceitação da versão em português da Motor Assessment Scale em pacientes com acidente vascular encefálico. Revista Brasileira de Fisioterapia, São Carlos, v. 13, n. 5, p. 405-411, 2009.

FARIAS, N.; BUCHALLA, C. M. A classificaçấo internacional de funcionalidade, incapacidade e saúde da organização mundial da saúde: conceitos, usos e perspectivas. Revista Brasileira de Epidemiologia, São Paulo, v. 8, n. 2, p. 187-193, 2005.

FERREIRO, K. N.; SANTOS, R. L.; CONFORTO, A. B. Psychometric properties of the Portuguese version of the Jebsen-Taylor test for adults with mild hemiparesis. Revista Brasileira de Fisioterapia, São Carlos, v. 14, n. 5, p. 377-382, 2010. 
GUILLEMIN, F; BOMBARDIER, C.; BEATON, D. E. Cross-cultural adaptation of health-related quality of life measures: litereture review and proposed guidelines. Journal of Clinical Epidemiology, Oxford, v. 46, n. 12, p. 1417-1432, 1993.

HUNTER, S. M.; CROME, P. Hand function and stroke. Reviews in Clinical Gerontology, London, v. 12, n. 1, p. 68-81, 2002.

LANGHORNE, P.; COUPAR, F.; POLLOCK, A. Motor recovery after stroke: a systematic review. Lancet Neurology, London, v. 8, n. 8, p. 741-754, 2009.

MAHER, C. G.; LATIMER, J.; COSTA, L. O. P. The relevance of cross-cultural adaptation and clinimetrics for physical therapy instruments. Revista Brasileira de Fisioterapia, São Carlos, v. 11, n. 4, p. 245-252, 2007.

MATHIOWETZ, V. et al. Adult norms for the box and block test of manual dexterity. American Journal of $\mathrm{Oc}$ cupational Therapy, Rockville, v. 39, n. 6, p. 386-391, 1985.

MICHAELSEN, S. M. et al. Confiabilidade da tradução e adaptação do Test d'Évaluation des Membres Supérieurs de Personnes Âgées (TEMPA) para o português e validação para adultos com hemiparesia. Revista Brasileira de Fisioterapia, São Carlos, v. 12, n. 6, p. 511-519, 2008.

MICHAELSEN, S. M. et al. Translation, adaptation and inter-rater reliability of the administration manual for the Fugl-Meyer assessment. Revista Brasileira de Fisioterapia, São Carlos, v. 15, n. 1, p. 80-88, 2011.

MORLIN, A. C. G. et al. Concordância e tradução para o português do Teste de Habilidade Motora do Membro Superior - THMMS. Revista Neurociências, São Paulo, v. 14, n. 2, p. 6-9, 2006.

ORGANIZAÇÃO MUNDIAL DE SAÚDE - OMS. Classificação internacional da funcionalidade, incapacidade e saúde. São Paulo: Edusp, 2003.
OXFORD, G. K. et al. Adult norms for a commercially available nine hole peg test for a finger dexterity. American Journal of Occupational Therapy, Rockville, v. 57, n. 5, p. 570-573, 2003.

PAZ, L. P. S.; BORGES, G. Teste da Ação da Extremidade Superior como medida de comprometimento após AVC. Revista Neurociências, São Paulo, v. 15, n. 4, p. 277-283, 2007.

PEREIRA, N. P. et al. Confiabilidade da versão brasileira do Wolf Motor Function Test em adultos com hemiparesia. Revista Brasileira de Fisioterapia, São Carlos, v. 15, n. 3, p. 257-265, 2011

PEREIRA, N. P. et al. Motor Activity Log-Brasil: confiabilidade e relaçôes com a função motora em indivíduos com hemiparesia crônica. Arquivos de Neuro-Psiquiatria, São Paulo, v. 70, n. 3, p.196-201, 2012.

POLIT, D. F.; BECK, C. T.; OWEN, S. T. Is the cvi an acceptable indicator of content validity? Appraisal and recommendations. Research in Nursing \& Health, New York, v. 30, n. 4, p. 459-467, 2007.

REICHENHEIM, M. E.; MORAES, C. L. Operacionalização de adaptação transcultural de instrumentos de aferição usados em epidemiologia. Revista de Saúde Pública, São Paulo, v. 22, n. 3, p. 1-9, 2007.

ROGER, V. L. et al. Heart disease and stroke statistics. Circulation, Dallas, v. 125, n. 1, p. e2-e220, 2012.

SCHUSTER, C.; HAHN, S.; ETTLIN, T. Objectively-assessed outcome measures: a translation and cross-cultural adaptation procedure applied to the Chedoke McMaster Arm and Hand Activity Inventory (CAHAI). BMC Medical Research Methodology, London, v. 10, n. 1, p. 106, 2010.

STREINER, D. L.; NORMAN, G. R. Health measurement scales: a practical guide to their development and used. New York: Oxford Medical Publications, 2003.

\section{Contribuição dos Autores}

Daniele Peres participou da concepção do estudo, da coleta e análise dos dados, e da redação do texto. Júlia Macruz Garcia participou da coleta e análise dos dados. Letícia Cardoso Rodrigues participou da análise dos dados, do comitê de especialistas e da revisão do manuscrito. Rodrigo José Knabben participou da coleta dos dados e da revisão do manuscrito. Fernanda Romaguera participou da tradução do manual e da revisão do manuscrito. Stella Maris Michaelsen é a pesquisadora responsável pela pesquisa e pela revisão do manuscrito. Todos os autores aprovaram a versão final do texto.

\section{Notas}

${ }^{1} \mathrm{O}$ artigo submetido faz parte da Dissertação de Mestrado da primeira autora, realizada no Programa de Pós-Graduação em Fisioterapia do Centro de Ciências da Saúde da Universidade do Estado de Santa Catarina - CEFID/UDESC. 